\title{
Reaction Time Measurement System for the Assessment of Persons with Auditory Processing Disorder
}

\author{
Hariprasad.B $^{1}$, Shreekanth.T ${ }^{2}$, Ajish.K.Abraham ${ }^{3}$ \\ ${ }^{1}$ (M.Tech Scholar, Industrial Electronics, Department of E\& CE, Sri Jayachamarajendra College of \\ Engineering, Mysore, India) \\ ${ }^{2}$ (Assistant Professor, Electronics and communication Department, Sri Jayachamarajendra College of \\ Engineering, Mysore, India) \\ ${ }^{3}$ (Professor and HOD, Department of Electronics, All India Institute of Speech and Hearing, Mysore, India)
}

\begin{abstract}
Auditory processing disorder (APD) is a set of problems that occur in different kinds of listening tasks. It is found in children and adults as well. Different methods are used in developed countries for the early detection of APD. Auditory processing disorder (APD) is a set of problems that occur in different kinds of listening tasks. It is found in children and adults as well. Different methods are used in developed countries for the early detection of APD. These methods are not advisable in testing children below 11 years. APD is directly related to the time taken by the person to react to a speech signal. In this paper the reaction time is measured using MATLAB program to find out whether a person can be tested for suspected APD.
\end{abstract}

Keywords: Auditory Processing Disorder, Reaction time, stimulus, cognition

\section{Introduction}

Communication issues and hearing problems have very high impact on the quality of life of a human being. Many people who report problems like difficulty in hearing in noisy environments, difficulty to understand speech when the speaker and way of speech is unfamiliar and reduced appreciation of listening to music etc. were treated for hearing related issues. Some of them even after the audiologic treatment continue to show the reported symptoms. This group of people, children and adults are a heterogeneous group of people who have difficulty in using auditory information to communicate and learn are said to have Auditory Processing Disorder (APD).

APD is not a specific problem or disease; rather it is a set of problems that occur in different kinds of listening tasks. Often children with APD are first diagnosed with attention deficit hyperactivity disorder (ADHD) or learning disabilities. APD refers to how the central nervous system uses auditory information.

Work in the field of recognizing APD is done at higher levels and normally children with APD are not recognized or treated for the problems they face. As a result these children will face a lot of difficulties related to their academics as well as day to day life.

Efforts are made in many parts of the world to find out an accurate method to detect APD. Most of them are conducted on clinical basis. Clinical methods like scanning and ear testing will not provide exact identification of APD since it is a set of problems that occur in different kinds of listening tasks. At present there exists some methods like selecting the correct order of pictures from a set of pictures and identifying a series of actions but such methods only address one among the many symptoms reported by the patient and did not give attention to the root cause of the problem. So far APD is not addressed from a technical point of view which will be easier and accurate to help doctors to identify persons with suspected APD from a group.

This paper approaches APD from a different angle. Auditory processing disorder refers to the processing problem occur in the central nervous system while using the auditory information. Purpose of the hearing mechanism is to hear the message conveyed through speech followed by comprehension. Comprehension leads to interpretation of the message by brain. In this mechanism cognition plays an important role. Cognitive ability is related to the brain functions of perception, recognition and interpretation of sensory stimuli like smell, touch and hearing. Cognitive development is the construction of thought processes including remembering, problem solving and decision making from childhood through adolescence to adulthood. APD has direct relation with cognitive abilities. When a person hear a speech signal which provides the information to perform some action, which is proportional to the reaction time taken by the nervous system to respond. A mathematical system which can be used to measure the reaction time for sound perception will be helpful for assessing and improving the cognitive skills of human beings especially the children.

This paper explains a mathematical model which represents the audio spectrum and shows the time difference between the offset of stimulus and onset of response by the listener. It is tested among a good sample 
and results obtained are compared. An algorithm is developed using which tests can be conducted in real time to identify a person with suspected APD.

\section{Existing APD Detection Methods}

APD is assessed through the use of special tests designed to assess the various auditory functions of the brain. However, before this type of testing begins, it is important that each person being tested receive a routine hearing test. An audiologist must rule out any "physical" hearing problems. The audiologist will assess the ability of the of the ear to detect a normal range of frequencies, from low tones to high tones, without loss of sensitivity. This referral is given to an associate Audiologist experienced in assessing children.

The auditory tests that are used to assess central auditory function fall into two major categories: Behavioral tests and Electrophysiological tests. It should be noted that children being assessed for APD will not necessarily be given a test from each of these categories. The selection of which test is used will depend upon a number of factors, including the age of the child, the specific auditory difficulties the child displays, the child's native language and cognitive status. Generally, children under the age of 7 years are not candidates for this type of diagnostic testing as neuromaturation of some portions of the auditory system may not be complete until age 12 or later. In addition, central auditory processing assessments may not be appropriate for children with significant developmental delays and cognitive deficits.

\subsection{Electrophysiological Tests}

Electrophysiological tests are measures of the brain's response to sounds. A special cap with built in sensors is placed on the head of the child for the purpose of measuring the electrical activity that arise from the central nervous system in response to an auditory stimulus. An auditory stimulus, often a clicking sound, or two tons of different frequencies are delivered to the child's ear and the electrical responses are recorded. Some frequencies evaluate processing low in the brain (auditory brainstem response), whereas others assess functioning higher in the brain (middle latency responses, late auditory evoked responses, auditory cognitive or P300 responses). The results obtained on these tests are compared to age-appropriate norms to determine if any abnormalities exist.

\subsection{Behavioral Tests}

Different types of behavioral tests are used. One such test is SCAN which is a screening test to find APD. SCAN is used to identify children who have auditory processing disorders and who may benefit from intervention. The test is administered to children ages 5 to 11 in approximately 20 minutes. Three subtests include low pass filtered words, auditory figure ground and competing words. SCAN A is another screening test for Auditory Processing Disorders in adolescents and adults was designed for individuals over 11 years. This instrument includes an additional subtest using competing sentences as stimuli.

\subsection{Monaural Low Redundancy Speech Tests}

Monaural low-redundancy speech tests represent a group of tests designed to test an individual's ability to achieve auditory closure when information is missing. The speech stimuli used in these tests have been modified by changing one or more of the following characteristics of the speech signal: frequency, timing (phase), or amplitude characteristics. The test items are presented to each ear individually and the child is asked to repeat the words that have been presented. A percent correct score is derived for each ear and these are compared to age-appropriate norms.

\subsection{Dichotic Speech Tests}

In these tests different speech items are presented to both ears either simultaneously or in an overlapping manner and the child is asked to repeat everything that is heard (divided attention) or repeat whatever is heard in one specified ear (directed attention). The more similar and closely acoustically aligned the test items, the more difficult the task.

\section{Drawbacks Of Available APD Tests}

All the above mentioned APD tests have some sort of drawbacks. Many behavioral tests of central auditory processing in current clinical use require a minimum developmental age of seven or eight years, or a level of cognitive functioning that is consistent with this age range. Electrophysiological tests are not advisable for small children in the age group of 5 to 10. The results invoked after the test are to be evaluated by an expert audiologist. Behavioral tests are used for children above 11 years and in adults. In the case of children in the 510 years age group where effect of APD is more behavioral tests are not used. Redundancy speech tests which require speech tests and group tests are also not useful in children. All these are language dependent and require the presence of audiologists. 
Dichotic speech tests are the most commonly used APD diagnostic test and can be used in children also. These type of score based systems will provide the result as whether a child can be treated for APD or not, but they have no mathematical basis in this process. Audiologists, related professionals, and clinical scientists generally agree that some of the tests for APD in current clinical use lack rigorous psychometric design, construction, and validation. Speech-language, psychological, and other tests cannot be used to diagnose APD, even if the term "auditory processing" is included in their titles or subtest descriptions. Concerns have been raised regarding the utility of tests normed on groups suspected of having APD for the identification of dysfunction of the central auditory pathways, with several clinical researchers arguing that their utility is limited at best.

The cost incurred in this process is high and in developed countries like USA, Canada and UK where reimbursement and insurance are easy to get these tests are common. In the case of developing countries like India where centralized reimbursement and medical insurance are out of reach for common people it is very difficult to conduct such tests.

\section{Methodology}

The method used to calculate the reaction time for the assessment of persons with auditory processing disorder using both oral and keyboard detection is explained in Fig 1.

The algorithm has been developed to estimate the time difference between the end point of stimulus of a speech signal and start point of a response signal. The speech signal used in this project is a vowel sound of very short duration recorded in a sound recording studio. This signal is in the .wav format. The response signals are of two types, oral and keyboard press. When the recorded signal is played through the earphone the child (or adult) who is to be tested has to respond to it by repeating what he heard. The two signals are recorded in the same file and the response time is measured using MATLAB. The algorithm for this is also developed. Keyboard pressing is another method used to find response time. The same sound is played from left or right side in a random manner and the child (or adult) has to respond it by pressing the left or right arrow key depending upon the direction from which the sound comes.

In both cases the response time is measured and then the resultant values are compared using another algorithm which will also include exact detection of the direction of sound. From this the response time comparison algorithm we can find out which child (or adult) among a particular group can be considered as suspected APD.

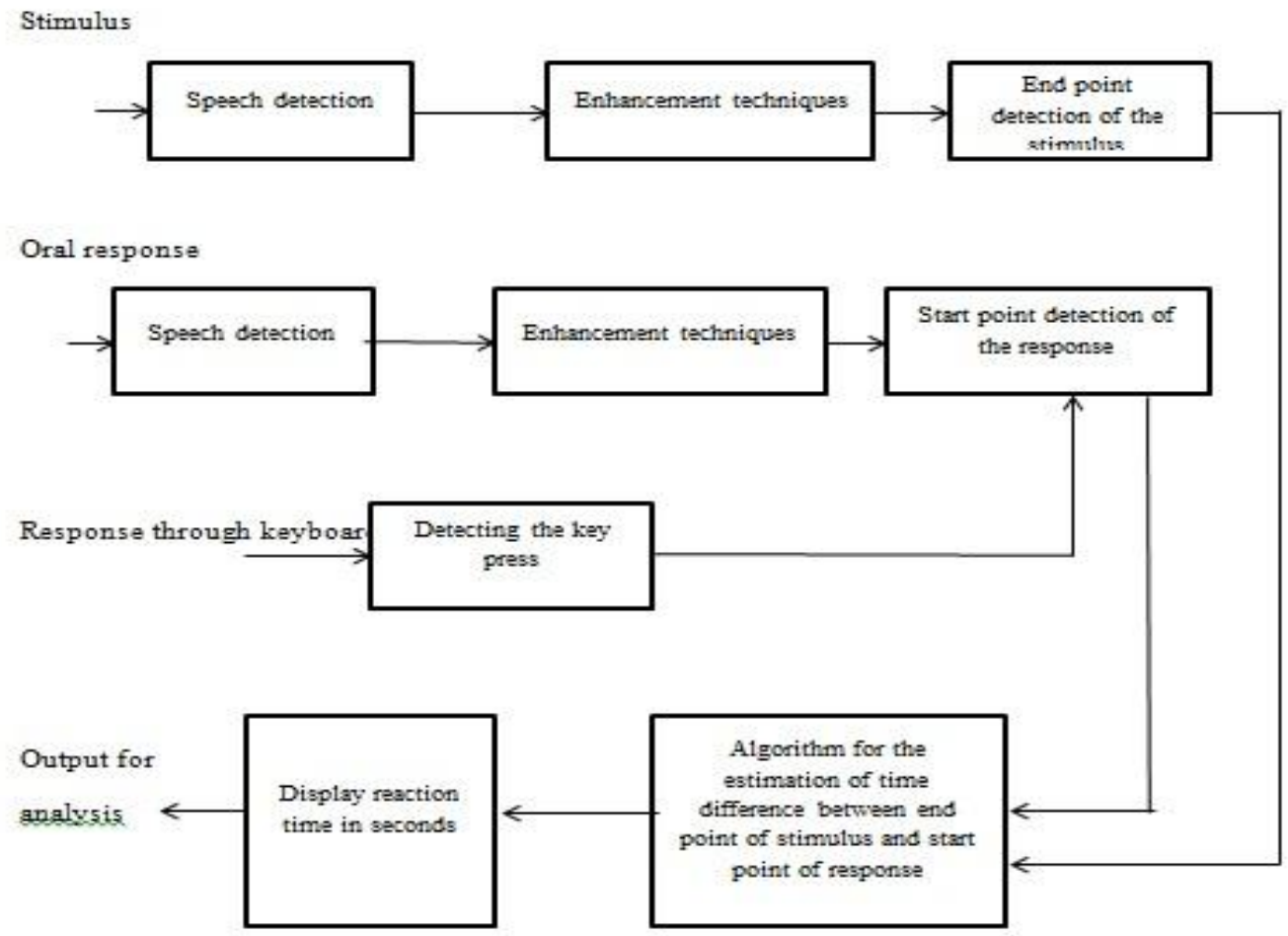

Fig 1. Block diagram 


\subsection{Method of calculation of reaction time for oral response using MATLAB}

In this project just an utterance is needed and vowels are used for this purpose. The vowel sounds " $\mathrm{A}$ " and "I" are recorded in a noise free studio which is used as the speech source. A group of children in the age group of 5-11 are selected as the target group. The recorded speech is played and these children are asked to repeat what they heard through a microphone. One by one the oral responses from all the children are recorded.

The stimulus is the speech signal representing "A" or "I". Detecting the end point of the stimulus is the first step in this project. The point at which the signal power becomes negligible is found out using MATLAB. This point is considered as the end point of the stimulus. A threshold value is assigned and when the signal amplitude falls below this value it is considered as its end point. From this end point onwards counting of the response time starts. The replay the child gives is also recorded in the same file. The point at which the child's sound becomes detectable the program stops counting the time. It is used as the start point of stimulus. Here also a threshold value is assigned. Time period up to this is measured in MATLAB. This period in temporal domain represents the time required for the child to make response, is called the response time. The speech signal and it's response in time domain is shown in Fig 2.

For this the speech and it's response are converted into digital format. The average value of 1000 samples is taken as a single stem which represents a positive vale as shown in Fig 3. This makes it easy to calculate the number of stems in the response time. From which the response time can be found by multiplying number of stems with 1000 .

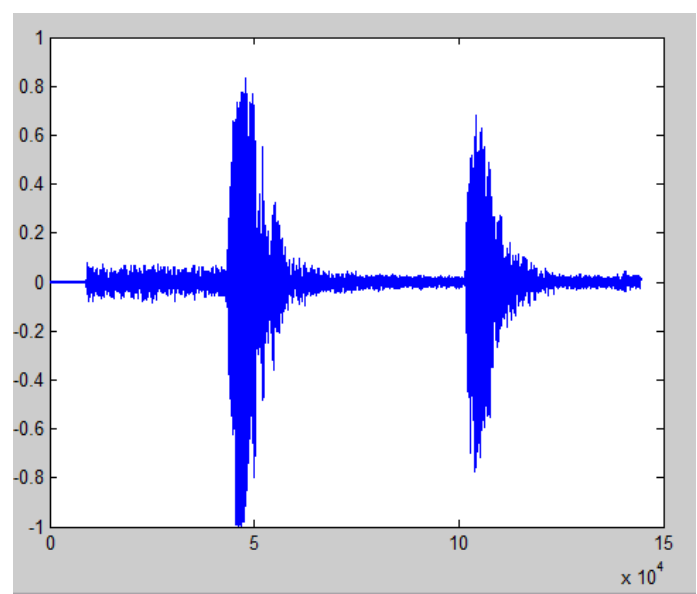

Fig 2 Time domain representation of speech

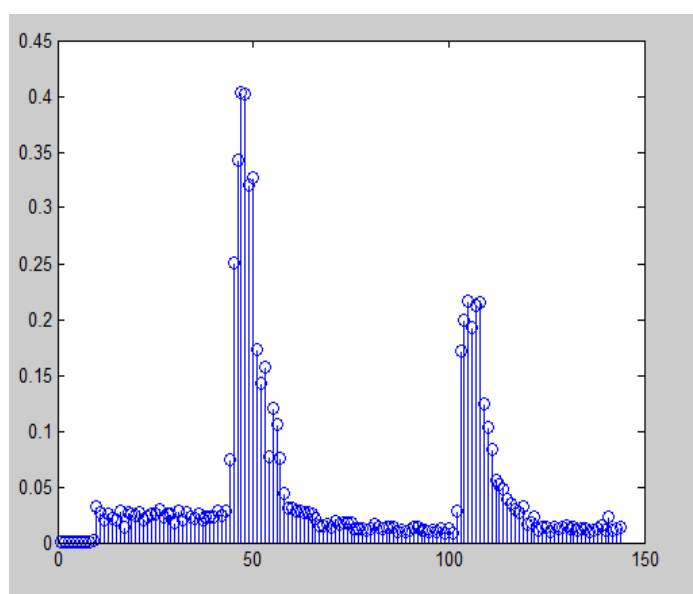

Fig 3 Digitized signal with stems

\subsection{Reaction time and direction detection using keyboard response}

The child is asked press the left or right keys when it hears the speech. Here the speech is produced through either of the two earphones randomly as in the case of oral response a threshold value is kept to classify children depending on the reaction time. The child has to find the direction of sound correctly and has to press the key before the threshold time.

\section{Results And Analysis}

In this project the reaction time of 10 children aged between 5 to 11 are taken as the target group. Both the tests are conducted and the results obtained are shown in Table 1

From the Table 1, it is found that the oral response time for the speech signal in a group of 10 children is in the range of $1.8 \mathrm{~s}$ to $2.83 \mathrm{~s}$. All except the child 5 responded within $2.5 \mathrm{~s}$. So in the response time algorithm the threshold value is set as 2.5 seconds. The child 5 whose response time is $2.83 \mathrm{~s}$ which is greater than $2.5 \mathrm{~s}$ can be tested for suspected APD. If the same threshold time is used in the keyboard testing also it is found that only the child 5 takes more than $2.5 \mathrm{~s}$ to respond. The accuracy in finding the direction of sound is $80 \%$ in the group since only 2 out of 10 children are wrong. 
Table 1

\begin{tabular}{|c|c|c|c|}
\hline Child No: & $\begin{array}{l}\text { Reaction time for } \\
\text { oral response } \\
\text { (seconds) }\end{array}$ & $\begin{array}{l}\text { Reaction time for } \\
\text { key press detection } \\
\text { (seconds) }\end{array}$ & $\begin{array}{c}\text { Whether } \\
\text { identified the } \\
\text { correct direction } \\
\text { of sound }\end{array}$ \\
\hline 1 & 2.22 & 2.02 & correct \\
\hline 2 & 1.83 & 2.39 & correct \\
\hline 3 & 1.79 & 2.32 & correct \\
\hline 4 & 1.9 & 2.14 & correct \\
\hline 5 & 2.83 & 2.41 & wrong \\
\hline 6 & 2.19 & 2.27 & correct \\
\hline 7 & 1.81 & 2.48 & correct \\
\hline 8 & 1.92 & 2.23 & correct \\
\hline 9 & 2.38 & 2.42 & wrong \\
\hline 10 & 1.8 & 2.15 & correct \\
\hline
\end{tabular}

In the keyboard response checking all expect two children responded correctly. The child 5 and child 8 were not able to find out the correct direction of sound but their response time for the keyboard was within the threshold limit which is set as 2.5 as in the case of oral response time measurement.

So as an observation of the APD detection test conducted among the group of 10 children, out of 10 only the child 5 can be tested for suspected APD. Among others child 8 has some hearing problems which may be related to suspect APD.

\section{Conclusion}

The new approach proposed in this paper to find the chances of Auditory Processing Disorder among children/adults is the first step in the development of an accurate and cost effective method to recognize and treat people having APD. The existing tests cannot be conducted on children of age less than 7, but the proposed test can be conducted in children at the age of 5. The algorithm developed here is useful to understand whether a child / adult can be included in a group who can be further tested for APD. Already it is tested on a group of 10 children and the results obtained are satisfactory.

This project with further development, once implemented will provide a cost effective method to identify children/ adults with APD in developing countries like India. Lack of awareness about APD among the people made them to undergo treatments for hearing loss which will not cure the symptoms. Once this system is implemented such problems can be solved. Portability is another advantage which makes the proposed system more useful.

\section{References}

[1]. Moore DR, Rosen S, Bamiou DE, Campbell NG, Sirimanna T. "Evolving concepts of developmental auditory processing disorder: A British society of Audiography. APD special interest group white paper". International journal Audiology. Jan 2013

[2]. Melanie Ferguson and Dave Moore. "Diagnosing APD Findings from a population study of auditory processing." MRC Institute of Hearing Research. Nottingham University. UK. 2008

[3]. Sonam Pal; M. Tech Scholar, EC, Department SSSIST, Sehore, India, Jaikaran Singh ; H.O.D and Associate Professor, EC Department, SSSIST, Sehore, India."Analytical Review of Feature Extraction Technique for Automatic Speech Recognition"International Journal of Science and Research (IJSR) NOV 2015

[4]. Nidhi Desai, Prof.Kinnal Dhameliya: Department of Electronics and communication engineering, CGPIT, Bardoli. Prof. Vijayendra Desai: Department Of Electronics and Communication Engineering, C.K.P.C.E.T., Surat."Recognizing voice commands for robot using MFCC and DTW": International Journal for advanced research in computer and communication engineering. May 2014, pp. $6456-6459$

[5]. Aseem Saxena, Amit Kumar Sinha, Shashank Chakravarthy and Surabhi Charu Suresh Gyan Vihar University, Jaipur, Rajasthan, India International Journal of Advances In Computer Science and Cloud Computing, ISSN: 2321-4058:Volume- 1, Issue- 2, Nov2013, pp.26-30 